\title{
The utility of flow sorting to identify chromosomes carrying a single copy transgene in wheat
}

\author{
Petr Cápal ', Takashi R. Endo 1,2, Jan Vrána ${ }^{1}$, Marie Kubaláková1, Miroslava Karafiátová ', Eva Komínková1, \\ Isabel Mora-Ramírez ${ }^{3}$, Winfriede Weschke ${ }^{3}$ and Jaroslav Doležel ${ }^{{ }^{*}}$
}

\begin{abstract}
Background: Identification of transgene insertion sites in plant genomes has practical implications for crop breeding and is a stepping stone to analyze transgene function. However, single copy sequences are not always easy to localize in large plant genomes by standard approaches.

Results: We employed flow cytometric chromosome sorting to determine chromosomal location of barley sucrose transporter construct in three transgenic lines of common wheat. Flow-sorted chromosomes were used as template for PCR and fluorescence in situ hybridization to identify chromosomes with transgenes. The chromosomes carrying the transgenes were then confirmed by PCR using DNA amplified from single flow-sorted chromosomes as template.

Conclusions: Insertion sites of the transgene were unambiguously localized to chromosomes 4A, 7A and 5D in three wheat transgenic lines. The procedure presented in this study is applicable for localization of any single-copy sequence not only in wheat, but in any plant species where suspension of intact mitotic chromosomes suitable for flow cytometric sorting can be prepared.
\end{abstract}

Keywords: Transgene localization, Flow cytometric sorting, Single chromosome amplification, Triticum aestivum, Hordeum vulgare, HvSUT1

\section{Background}

During the past 30 years, many cultivars of agricultural crops beneficial to humankind have been developed by means of genetic engineering, including plants resistant to herbicides, pests or viruses, bearing fruits with prolonged shelf life and products more suited for industrial processing [for review see 1]. Wheat ranks 5 th in the commodities produced worldwide and is the second most-produced food crop occupying more than $50 \%$ of the world crop area (http://faostat3.fao.org/). In the light of climate change and world population growth, future challenges for the increase of crop production have constantly been discussed. However, FAO statistics show that

\footnotetext{
${ }^{*}$ Correspondence: dolezel@ueb.cas.cz

${ }^{1}$ Institute of Experimental Botany, Centre of the Region Haná for Biotechnological and Agricultural Research, Šlechtitelů 31, 78371 Olomouc, Czech Republic

Full list of author information is available at the end of the article
}

the wheat production is reaching a plateau and is severely affected by climate change. This is a consequence of a slowdown in wheat yield increase, accounting for only $0.5 \%$ per year in the last decade [2].

Breeding improved cultivars with increased tolerance to adverse climatic conditions and with increased yield and quality could be facilitated by genetic engineering and introduction of beneficial genes from other organisms. The insertion site of a transgene is of great importance for the transgene function [3, 4] which is also influenced by its position on the chromosome, including the flanking DNA sequences [5]. However, transgene localization is not easy by routine approaches, like fluorescence in situ hybridization (FISH), or Southern blotting. A prevalent method for detection of transgenes in animals and plants is FISH, which has its pros and cons [6]. In barley and common wheat, FISH enables cytological localization of cDNAs, as short as $1.5 \mathrm{~kb}$, on a 
chromosome or chromosomes that had already been known to carry the cDNAs $[7,8]$. Although some authors succeeded in localizing transgenes on plant chromosomes using FISH [9-11], this approach has not become a routine application.

Weichert et al. [12] obtained transgenic lines (HOSUT) of hexaploid wheat carrying barley (Hordeum vulgare) sucrose transporter HvSUT1 (SUT) gene that is overexpressed under the control of the endosperm-specific Hordein B1 promoter $(\mathrm{HO})$. The HOSUT lines were found to increase grain yield significantly as compared to control non-transformed plants [13]. However, the genomic location of the transgene in these lines was not known. In the present work we employed a novel approach for unambiguous identification of chromosomes carrying the transgene in three HOSUT lines. The protocol takes the advantage of the availability of a procedure for flow cytometric chromosome sorting in wheat and the fact that flow-sorted chromosomes are suitable as templates for PCR and FISH [14]. Moreover, a protocol has been developed recently for representative DNA amplification from single copies of chromosomes [15]. By combining these approaches we could assign the transgene to particular chromosomes in three HOSUT lines of wheat.

\section{Results and discussion}

The experimental workflow is shown on Fig. 1. As the first step, we prepared liquid suspensions of intact mitotic chromosomes from all five lines of wheat (see "Methods" section) and analyzed them by flow cytometry. Monovariate flow karyotypes (histograms of relative fluorescence intensity) were obtained after the analysis of DAPI-stained chromosomes, and bivariate flow karyotypes obtained after the analysis of DAPI-stained chromosomes with FITC-labelled GAA microsatellites. We observed differences between flow karyotypes of the HOSUT lines and the model hexaploid wheat cultivar Chinese Spring. The alterations concerned the profiles of major composite peaks on monovariate flow karyotypes (Additional file 1: Figure S1) and the distribution of chromosome populations on bivariate flow karyotypes (Fig. 2). This observation reflected the differences in karyotypes (chromosome polymorphism) between the cultivar Certo, used to produce the HOSUT lines (data not shown), and Chinese Spring. On the other hand, flow karyotypes of the three HOSUT lines were indistinguishable from each other.

In order to identify chromosomes carrying the transgenes, we first used the approach described by Vrána et al. [16]. Fractions of 200 chromosomes were sorted from different regions of monovariate flow karyotypes as shown on Additional file 1: Figure S1, and
DNA of the sorted chromosomes was used as template for PCR. This analysis identified one region (sort gate) in each line as representing chromosomes bearing a transgene. As each region (sort gate) on a monovariate flow karyotype may represent more than one chromosome type, in the next step we sorted chromosomes from regions delineated on bivariate flow karyotypes (Fig. 2). The sort gates were designed to include chromosome populations corresponding to the positive sort gates on monovariate flow karyotypes. From these regions, and also from nearby regions, chromosomes were sorted into PCR tubes (100 chromosomes per tube) and immediately afterwards also onto microscopic slides (ca. 1000 chromosomes per slide). The results obtained by PCR with primers amplifying HvSUT-RT sequence (Fig. 3) and identification of chromosomes from sort gates for each transgenic line by FISH with probes targeting Afa-repeat family and GAA-microsatellites (Fig. 4) are summarized in Table 1.

FISH analysis showed that more than $90 \%$ of chromosomes flow-sorted from the region defined by the green rectangle consisted of one type of chromosome in each of the HOSUT lines. This fact together with the results of PCR suggested that the transgene was located on chromosome 7A in HOSUT 12/44, on chromosome 5D in HOSUT 20/6 and on chromosome 4A in HOSUT 24/31. In the former two lines, the critical type of chromosome was not found among the chromosomes flow-sorted from the region defined by red rectangles. However, chromosome $4 \mathrm{~A}$ was found to represent $12.39 \%$ of chromosomes flow-sorted from the red region in HOSUT $24 / 31$. This was probably due to the similarities in size and the amount of GAA-FITC fluorescence of chromosomes $4 \mathrm{~A}$ and $7 \mathrm{~A}$. Due to this similarity, mixture of the two chromosomes $4 \mathrm{~A}$ and $7 \mathrm{~A}$ was also observed in the chromosome fraction sorted from the green region in HOSUT 12/44.

To confirm chromosomal locations of the transgene and avoid ambiguous results due to possible contamination of flow-sorted fraction by other chromosomes, PCR was done on DNA amplified from single flow-sorted chromosomes. As each time only one copy of chromosome is sorted, the DNA cannot be contaminated by other chromosomes. Five single chromosomes were sorted from the green sort regions of the HOSUT lines and their DNA was separately amplified using multiple displacement amplification (MDA). Out of the five sorted chromosomes, whole genome amplification was successful with three chromosomes in HOSUT 12/44, two chromosomes in HOSUT 20/6 and four chromosomes in HOSUT 24/31. The successful amplification was defined by the production of measurable amount of DNA after MDA and by the presence of at least one marker for the 


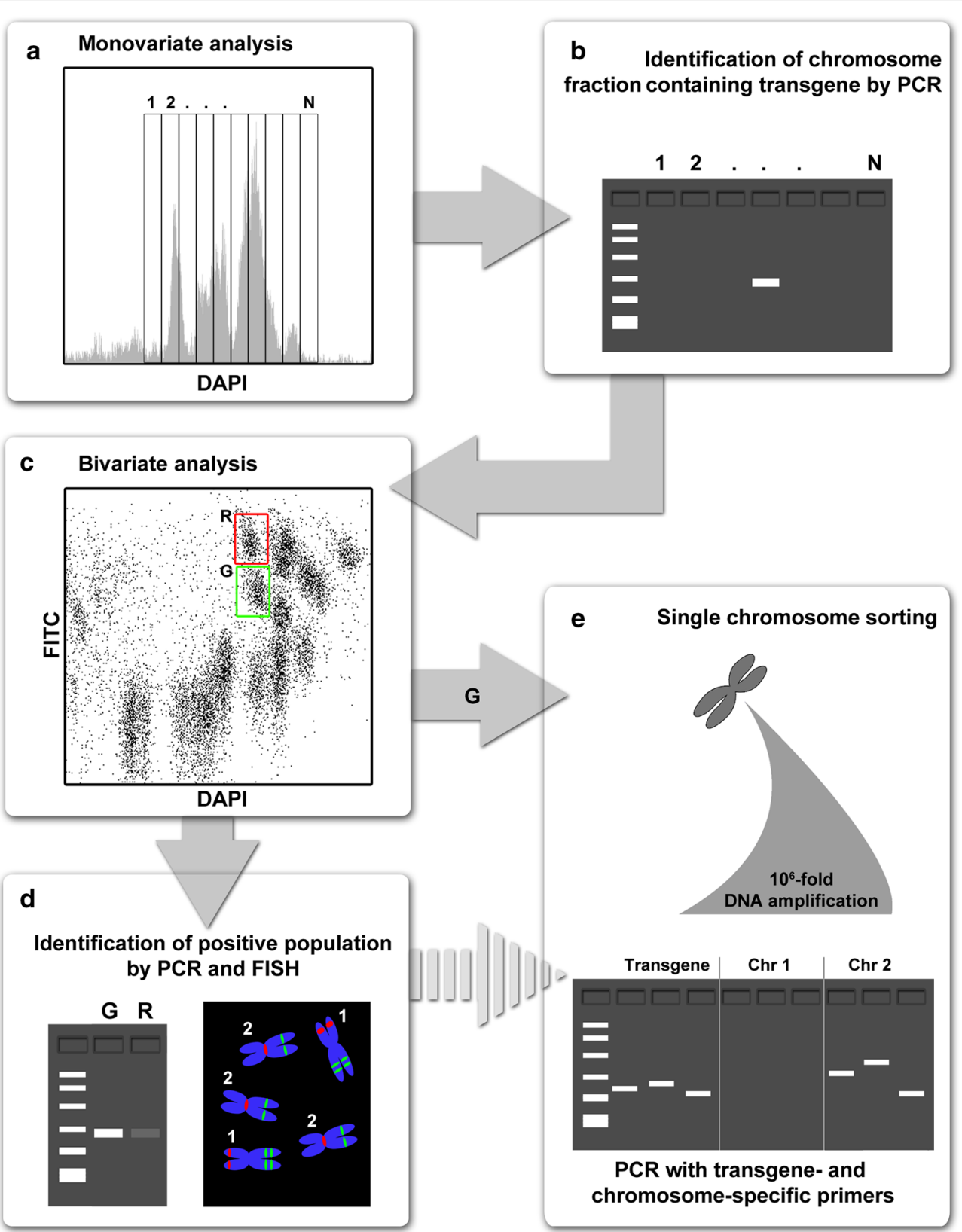

Fig. 1 Experimental workflow. a Monovariate flow karyotype is dissected into small regions. From each region, 200 chromosomes are sorted. b Flow-sorted chromosomes are used as template for PCR with transgene-specific marker. c The region representing chromosome with the transgene identified on monovariate flow karyotype is dissected by sorting chromosomes from sort regions on bivariate flow karyotype. $\mathbf{d}$ From the sort gates on the bivariate flow karyotype, 100 chromosomes are sorted for PCR with transgene-specific marker. Moreover, 1000 chromosomes are sorted immediately afterwards onto the microscopic slides to identify flow-sorted chromosomes by FISH. e From the sort gate most enriched for the transgene-bearing chromosome (region $G$ in this example), single chromosomes are sorted individually into PCR tubes. DNA of single-flow sorted chromosomes is amplified and resulting DNA is used as template for PCR to identify the presence of multiple transgene- and chromosome-specific sequences. This step unambiguously confirms the chromosome with integrated transgene

transgene and one marker for the wheat chromosome. The reason for occasional failure to amplify DNA from single chromosomes, which was observed previously [15] is not clear. One explanation is that a droplet with sorted chromosome lands on side wall of PCR tube and the chromosome is excluded from the MDA reaction. The amount of chromosomal DNA in successfully amplified samples ranged from 0.3 to $1.7 \mu \mathrm{g}$ DNA.

Chromosome specificity of sequence tagged site (STS) markers used in this work to identify individual 

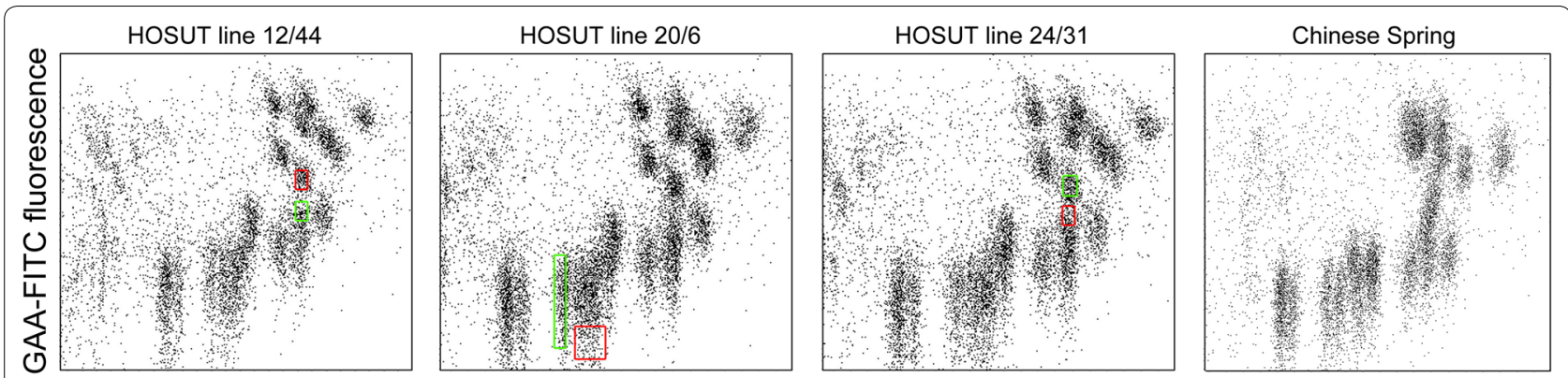

\section{DAPI fluorescence}

Fig. 2 Bivariate flow karyotypes of three transgenic HOSUT lines of wheat obtained after the analysis of chromosomes with FITC-labelled (GAA) microsatellites and stained by DAPI. The position of red and green regions used to sort particular chromosomes is indicated. The green sort gate was found to represent chromosomes carrying transgene. Chromosomes were flow-sorted also from the neighboring population delineated by red gate and were used as a control. Although the transgene-bearing chromosome should not be included in this region, the sorted population could potentially be contaminated with transgene-bearing chromosomes due to similarity in chromosome size and DNA content

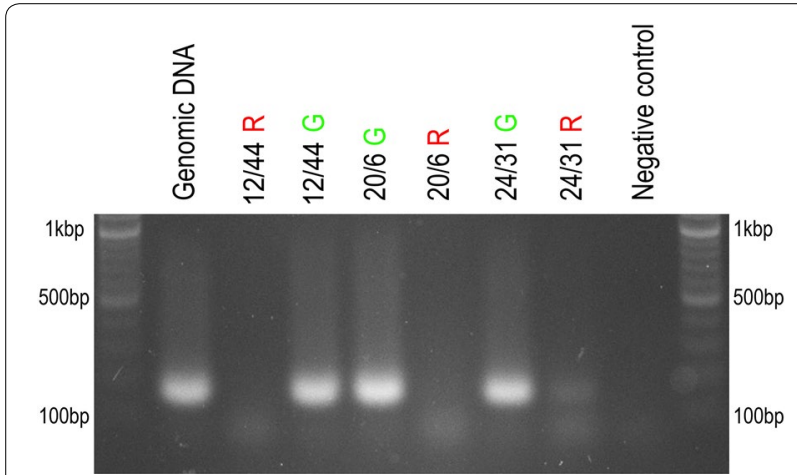

Fig. 3 Agarose gel electrophoresis of PCR products obtained with primers for the transgene and DNA of chromosomes flow-sorted from three HOSUT lines using the green and red sort regions as shown in Fig. 1. The amplicon of HvSUT-RT (169 bp) was obtained with chromosomes sorted from the green sort region in all three HOSUT lines. When chromosomes were sorted from the red sort regions, no PCR amplification occurred for HOSUT 12/44 and HOSUT 20/6. However, a weak band was observed for HOSUT 24/31. Genomic DNA of the transgenic lines served as positive control chromosomes was first tested using the euploid and corresponding nulli-tetrasomic lines of Chinese Spring (Additional file 2: Figure S2). The results confirmed that the markers were suitable for unambiguous identification of wheat chromosomes $1 \mathrm{~A}, 4 \mathrm{~A}, 5 \mathrm{D}$ and $7 \mathrm{~A}$. PCR analysis using both transgene- and chromosome-specific markers clearly confirmed chromosome location of transgenes as determined in the first part of this study. In case of HOSUT 24/31, where the location of the transgene was ambiguous, all four transgene markers were detected in DNA amplified from single chromosomes sorted from the green region (Fig. 5), and all four 4A-specific markers were also amplified in the same amplicons. None of the four 7A-specific markers was found in the same amplicons.

\section{Conclusions}

Coupling PCR and FISH mapping using flow-sorted mitotic chromosomes as templates narrowed down the list of candidate chromosomes harboring the transgene to one or two chromosomes. PCR on DNA amplified
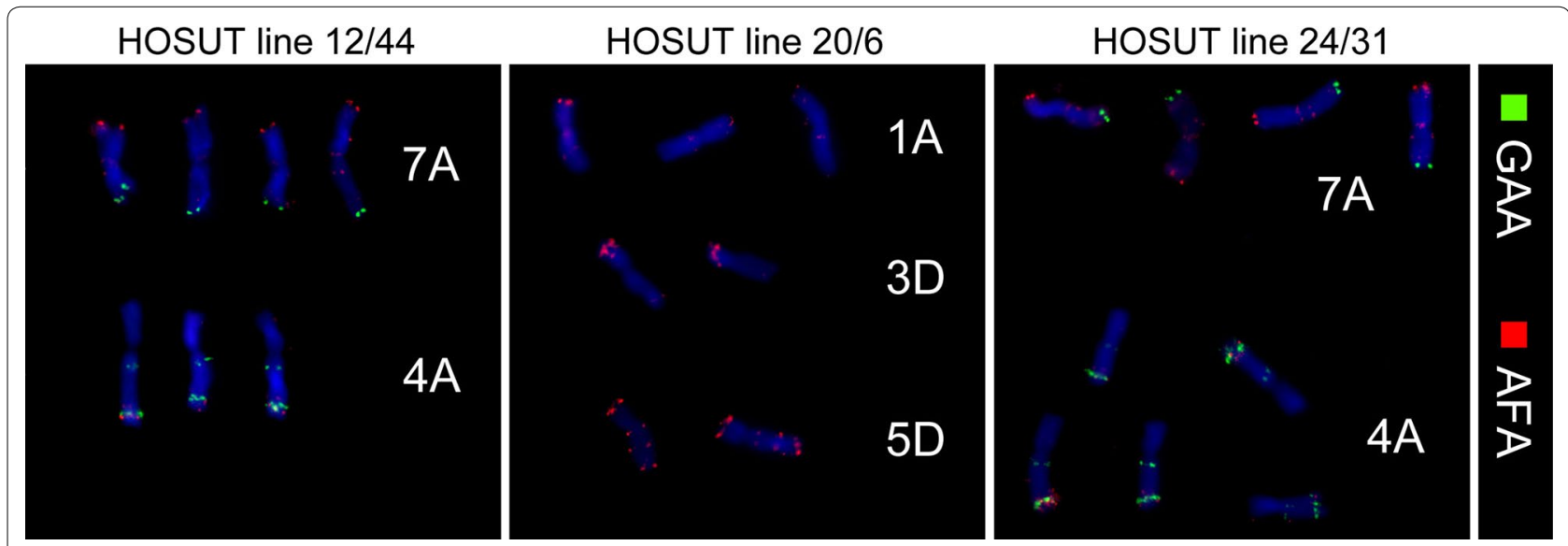

Fig. 4 Representative images of chromosomes flow-sorted from three HOSUT lines using the green and red sort regions on bivariate flow karyotypes as shown in Fig. 2. FISH was done using probes for Afa-family (red signals) and GAA microsatellites (green signals). Chromosomes were counterstained with DAPI (blue) 
Table 1 PCR and FISH analysis of chromosomes sorted from each of the sort gates in three HOSUT lines

\begin{tabular}{llll}
\hline Transgenic line & Sort gate & PCR result & $\begin{array}{l}\text { Chromosomes } \\
\text { identified FISH }\end{array}$ \\
\hline HOSUT 12/44 & Red & Negative & 4A (92.65\%) \\
& Green & Positive & 7A (90.90\%) \\
& & & $4 \mathrm{~A}(4.45 \%)$ \\
HOSUT 20/6 & Red & Negative & 1A (63.75\%) \\
& & & $3 \mathrm{D}(36.25 \%)$ \\
& Green & Positive & 5D (94.66\%) \\
& & & 1A (5.33\%) \\
HOSUT 24/31 & Red & Semi-positive & 7A (83.19\%) \\
& & & $4 \mathrm{~A}(12.39 \%)$ \\
& & & 2A (4.42\%) \\
& Green & Positive & 4A (97.30\%) \\
\hline
\end{tabular}

a Sort gates delineated with green and red rectangles in Fig. 2

b A faint band was visible after agarose gel electrophoresis of PCR product

c More than 1000 chromosomes were examined in each sorted fraction in each line

from single flow-sorted chromosomes then unambiguously identified the chromosomes with the integrated transgene. If chromosome-specific PCR-based markers are available, mapping on single copy chromosomes could be an ultimate approach to assign single copy DNA sequences, including transgenes, to particular chromosomes. Moreover, the sequence assembly of amplicons from the chromosome could allow detecting the position of transgene insertion, if enough sequence information on the chromosome is available. However the main purpose of this work was to assign a transgene to particular chromosomes. The approach presented here is currently applicable to more than 25 plant species, which include important cereals and legumes [14] where liquid suspensions of mitotic chromosomes suitable for flow cytometric sorting can be prepared.

\section{Methods \\ Plant material}

We used German winter wheat cultivar Certo (Triticum aestivum $\mathrm{L}$., $2 \mathrm{n}=6 \mathrm{x}=42$, genome formula AABBDD) and its three transgenic lines, HOSUT 12/44, HOSUT $20 / 6$ and HOSUT 24/31. The transgenic lines contain a single copy of the HvSUT1-cDNA (1894 bp) fused to the barley HorB1 promoter (550 bp) and the barley HorB1 terminator (1663 bp) [12]. We also used euploid and nullisomic-tetrasomic (Nt1A1B, Nt7A7B, Nt5D5B) lines of hexaploid wheat cultivar Chinese Spring (obtained from NBRP-wheat) to confirm the specificity of PCR markers to particular wheat chromosomes.

\section{Flow cytometric chromosome sorting}

Cell cycle synchronization and metaphase accumulation of root tip meristem cells was performed as described

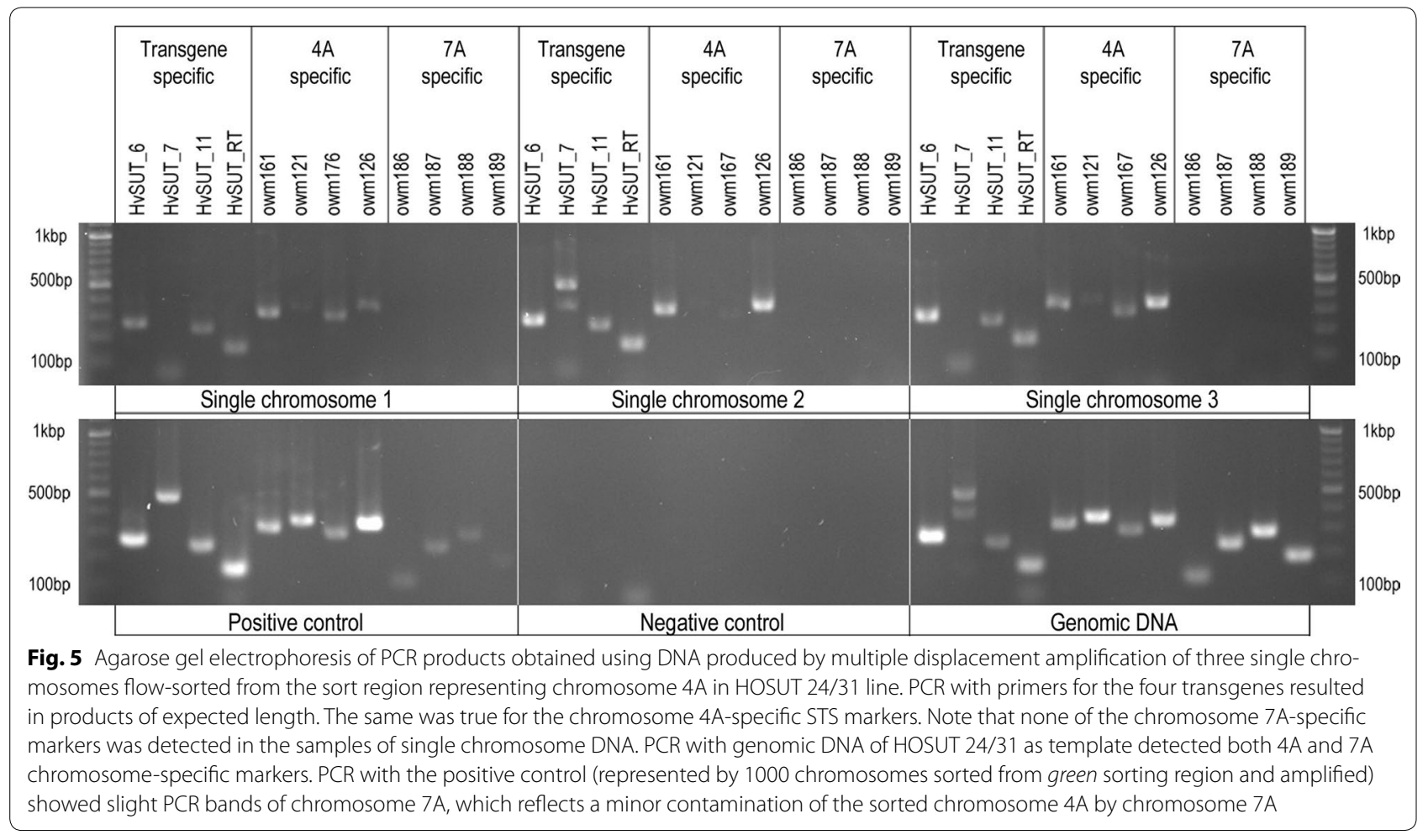


previously [17], except for the formaldehyde fixation, which was shortened to $15 \mathrm{~min}$. Isolated chromosomes were labelled by FISHIS (fluorescence in situ hybridization in suspension) using FITC-labeled GAA probe following the protocol of Giorgi et al. [18]. Flow cytometric analysis and sorting was done on BD FACSAria II high speed flow sorter equipped with $390 \mathrm{~nm}$ laser for DAPI excitation and $488 \mathrm{~nm}$ laser for FITC excitation. Sort gates were initially drawn on monovariate flow karyotypes of DAPI fluorescence (not shown) and subsequently on bivariate flow karyotypes of DAPI fluorescence versus GAA-FITC fluorescence as shown in Fig. 1.

\section{Fluorescence in situ hybridization (FISH)}

For microscopic observations, 1000 chromosomes were sorted onto microscope slides from each of the sort regions. The slides were left to air-dry in the dark overnight. Then the preparations were used for FISH following the protocol of Kubaláková et al. [19] using a Cy5-labeled probe targeting Afa-family repeats, the chromosomes were already labeled by a GAA microsatellite probe during the FISHIS procedure.

\section{PCR}

PCR was done using primers specific for the HOSUT transgene and for markers specific for candidate wheat chromosomes (Table 2). Of the four HOSUT primers, three were designed in the HvSUT1 region (accession no. AJ272309) and one in the HorB1 terminator region (accession no. FN643080). Wheat chromosome-specific markers were designed by Primer3 based on the chromosome sequences from the International Wheat Genome Sequencing Consortium (IWGSC), while preventing the primers from amplifying the sequence from the homoeologous chromosomes. PCR conditions were set as follows: initial denaturation $95^{\circ} \mathrm{C}$ for $3 \mathrm{~min}, 35$ cycles of $30 \mathrm{~s}$ denaturation at $95{ }^{\circ} \mathrm{C}$, annealing at $58-62{ }^{\circ} \mathrm{C}$ (see Table 2 for $\mathrm{T}_{\mathrm{a}}$ of the primer pairs) for $30 \mathrm{~s}$ and extension at $72{ }^{\circ} \mathrm{C}$ for $30 \mathrm{~s}$, followed by final extension at $72{ }^{\circ} \mathrm{C}$ for $5 \mathrm{~min}$. The amount of template DNA was $5 \mathrm{ng}$ for each reaction. PCR

Table 2 List of PCR primers for the HOSUT transgene construct and PCR primers for wheat STS markers on chromosomes 1A, 4A, 7A and 5D

\begin{tabular}{|c|c|c|c|c|c|}
\hline Name & Target & Forward primer sequence & Reverse primer sequence & $\begin{array}{l}\text { Amplicon } \\
\text { size (bp) }\end{array}$ & $\begin{array}{l}\text { Annealing } \\
\text { temperature }\left({ }^{\circ} \mathrm{C}\right)\end{array}$ \\
\hline HvSUT_6 & HOSUT1 & AGCGGCGGCGGTCACTGACTG & CCAAAGGACGACACCCCAGCC & 265 & 62 \\
\hline HvSUT_7 & HorB1 terminator & ATTAATTCCTCCCCGACCCTGC & CAATGGAGACGGCGCGTGCAA & 471 & 62 \\
\hline HvSUT_11 & HOSUT1 & GGCGGAACCCGCCGTGCAG & CCTGCGTCTTCCCCATCTGGAAGTA & 241 & 62 \\
\hline HvSUT_RT & HOSUT1 & CGGGCGGTCGCAGCTCGCGTCTATT & CATACAGTGACTCTGACCGGCACACA & 169 & 62 \\
\hline Owm121 & Chromosome 4A & ATTGCCGTCGCGAACTAGA & CGGGACGAGCTTGACGAT & 351 & 60 \\
\hline Owm126 & Chromosome 4A & CCAGTCAGAAATTATTATGAACCTATC & CGCTGTCTCGAGATTGGAGT & 342 & 60 \\
\hline Owm161 & Chromosome 4A & TTTTCAAGCAGGTTTTGTGC & TCACTTCTCTTCTTTGCGTTCA & 324 & 60 \\
\hline Owm167 & Chromosome 4A & TTTTCTTGGTCAGTATAACCTGTTTTT & TGAGCAGAGAAAAATTTCCAAG & 285 & 60 \\
\hline Owm174 & Chromosome 1A & GCATCCTAGTTTCTCTCTCAAGT & AACAAGATCACGAGCGAATTG & 157 & 58 \\
\hline Owm175 & Chromosome $1 \mathrm{~A}$ & AAACCCCTGATACTCATGCG & GTTTCTTGTCATTCATGTCACTTGT & 530 & 58 \\
\hline Owm176 & Chromosome $1 \mathrm{~A}$ & TTCCTGTCTGACTCCGCG & AACCACAACCGTCAACCG & 104 & 58 \\
\hline Owm177 & Chromosome $1 \mathrm{~A}$ & GTAGTCTGCTCCCGAGGAAT & GTCTCTAACCATACATCCATGAAGT & 192 & 58 \\
\hline Owm178 & Chromosome $1 \mathrm{~A}$ & CAACTTCTTCACATCCCGGAA & ATTTGGCCCTATGAGATATAATTACG & 306 & 58 \\
\hline Owm179 & Chromosome 1A & ACACTGTGATACCTCTAGATGTATG & CACATTGCCTATAAATTCTAAAAGGTC & 425 & 58 \\
\hline Owm180 & Chromosome 5D & CGGACGAGCAGCAGTACC & GCAGATCGGCATAAATTGAATGT & 292 & 58 \\
\hline Owm181 & Chromosome 5D & GGAGGTGTTCTAGGTGTACTTACT & AGAGCAATGTCAGAAGTCATCG & 240 & 58 \\
\hline Owm182 & Chromosome 5D & TCTCCACCTGCAGAGTCG & CATCAGGCCACAGTGTCAAT & 119 & 58 \\
\hline Owm183 & Chromosome 5D & TGTCCACACATTTCCCGTATG & AGTGGTGGATGTGGTTGCT & 196 & 58 \\
\hline Owm184 & Chromosome 5D & AGCATGCTCCCAAAGACTATTAC & GTTATGATGGTGGTAGCAATTTGA & 400 & 58 \\
\hline Owm185 & Chromosome 5D & GTGAACCTATATGACATCTTACCGG & GGGGCAGTTGTCAAGTATTGC & 421 & 58 \\
\hline Owm186 & Chromosome 7A & СTCTCTGTGGCCAATAGTGC & TCTATACCTCAACCCTACATCCA & 112 & 58 \\
\hline Owm187 & Chromosome 7A & GGCCACGAATTCCACAAGTA & CTATCGATCAACCAACCATCCA & 229 & 58 \\
\hline Owm188 & Chromosome 7A & GTACGAGTGCAGACAGTGTG & ACAATTAATTATACGCCCAGTTAAGC & 282 & 58 \\
\hline Owm189 & Chromosome 7A & CGTGCTTTCTTCTTCCTCCG & GCAGGTTAGTTTCTTGTGGTTG & 185 & 58 \\
\hline Owm190 & Chromosome 7A & CGCATGGACATTGTTCTAGTCA & GCACTTAGGCACGCTTGAG & 517 & 58 \\
\hline Owm191 & Chromosome 7A & CGACGACATTAGGAATATGGGAT & TGCGTGTGGGTGTGCTTA & 402 & 58 \\
\hline
\end{tabular}


products were run on $1.5 \%$ agarose gels. PCR using 100 sorted chromosomes as template was conducted after a few freeze-thaw cycles to disintegrate the chromosomes and the initial denaturation step was prolonged for $7 \mathrm{~min}$.

\section{Whole genome amplification of single chromosomes} DNA amplification of single chromosomes was performed by MDA using a GE Healthcare GenomiPhi V2 kit (GE Healthcare Life Sciences, Little Chalfont, UK) according to Cápal et al. [15]. Five individual chromosomes were flow-sorted into five $0.2 \mathrm{ml}$ PCR tubes from green sort gates from each HOSUT line and their DNA amplified. The amplified DNA was evaluated on $1.5 \%$ agarose gel, purified using magnetic beads (AMPure XP system, Beckman Coulter, Inc., Brea, CA, USA) and the concentration was measured by a spectrophotometer (NanoDrop, Thermo Fisher Scientific Inc., Waltham, MA, USA).

\section{Additional files}

Additional file 1: Figure S1. Flow karyotypes (histograms of fluorescence intensity) obtained after the analysis of DAPI-stained chromosomes isolated from three transgenic lines and cv. Chinese Spring of common wheat. Flow karyotypes of the transgenic lines are indistinguishable from each other, and slightly differ in profiles of the major composite peaks from those of Chinese Spring.

Additional file 2: Figure S2. Verification of marker specificity. PCR with a full set of chromosome-specific wheat STS markers was performed using genomic DNA of cv. Chinese Spring and corresponding nullitetrasomic lines for chromosomes 1A, 4A, 5D and 7A. The markers, which resulted in amplification products only in Chinese Spring and not in the nullitetrasomic lines, were used in this study.

\section{Authors' contributions}

The study was conceived and designed by $T E, P C$ and JD, experiments were performed by PC, TE, M Ka, M Ku, JV, IMR and EK, manuscript was written by $P C, T E, J D$ and WW. All authors read and approved the final manuscript.

\section{Author details}

${ }^{1}$ Institute of Experimental Botany, Centre of the Region Haná for Biotechnological and Agricultural Research, Šlechtitelů 31,78371 Olomouc, Czech Republic. ${ }^{2}$ Faculty of Agriculture, Ryukoku University, 1-5 Yokotani, Seta Oe-cho, Otsu, Shiga 520-2194, Japan. ${ }^{3}$ Leibniz Institute of Plant Genetics and Crop Plant Research (IPK) Gatersleben, Corrensstrasse 3, 06466 Stadt Seeland, Germany.

\section{Acknowledgements}

We thank Zdeňka Dubská for technical assistance with flow cytometric chromosome sorting. This work was supported by the National Program of Sustainability (Award No. LO 2014) and the Czech Science Foundation (Award No. P501-12-G090).

\section{Competing interests}

The authors declare that they have no competing interests.

Received: 20 February 2016 Accepted: 19 April 2016

Published online: 25 April 2016

\section{References}

1. Ahmad P, Ashraf M, Younis M, Hu X, Kumar A, Akram NA, Al-Qurainy F. Role of transgenic plants in agriculture and biopharming. Biotechnol Adv. 2012;30:524-40.

2. Fischer RA. The importance of grain or kernel number in wheat: a reply to Sinclair and Jamieson. Field Crops Res. 2008;105:15-21.

3. Svitashev S, Anaiev E, Pawlowski WP, Somers DA. Association of transgene integration sites with chromosome rearrangements in hexaploid oat. Theor Appl Genet. 2000;100:872-80.

4. Kumar S, Fladung M. Gene stability in transgenic aspen (Populus). II. Molecular characterization of variable expression of transgene in wild and hybrid aspen. Planta. 2001;213:731-40.

5. Kooter JM, Matzke A, Meyer P. Listening to the silent genes: transgene silencing, gene regulation and pathogen control. Trends Plant Sci. 1999:4:340-7.

6. Svitashev KS, Somers DA. Characterization of transgene loci in plants using FISH: a picture is worth a thousand words. Plant Cell Tiss Org Cult. 2002;69:205-14.

7. Danilova TV, Friebe B, Gill BS. Development of a wheat single gene FISH map for analyzing homoeologous relationship and chromosomal rearrangements within the Triticeae. Theor Appl Genet. 2014;127(3):715-30.

8. Karafiátová M, Bartoš J, Kopecký D, Ma L, Sato K, Houben A, Doležel J. Mapping nonrecombining regions in barley using multicolor FISH. Chromosome Res. 2013;21:739-51.

9. Pedersen C, Zimny J, Becker D, Jähne-Gärtner A, Lörz H. Localization of introduced genes on the chromosomes of transgenic barley, wheat and triticale by fluorescence in situ hybridization. Theor Appl Genet. 1997;94:749-57.

10. Dong J, Pushpa K, Cervera M, Hall TC. The use of FISH in chromosomal localization of transgenes in rice. Methods Cell Sci. 2001;23:105-13.

11. Khrustaleva LI, Kik C. Localization of single-copy T-DNA insertion in transgenic shallots (Allium cepa) by using ultra-sensitive FISH with tyramide signal amplification. Plant J. 2001;25:699-707.

12. Weichert N, Saalbach I, Weichert H, Kohl S, Erban A, Kopka J, Hause B, Varshney A, Sreenivasulu N, Strickert M, Kumlehn J, Weschke W, Weber $H$. Increasing sucrose uptake capacity of wheat grains stimulates storage protein synthesis. Plant Physiol. 2010;152:698-710.

13. Saalbach I, Mora-Ramírez I, Weichert N, Andersch F, Guild G, Wieser H, Koehler P, Stangoulis J, Kumlehn J, Weschke W, Weber H. Increased grain yield and micronutrient concentration in transgenic winter wheat by ectopic expression of a barley sucrose transporter. J Cereal Sci. 2014;60:75-81.

14. Doležel J, Vrána J, Cápal P, Kubaláková M, Burešová V, Šimková H. Advances in plant chromosome genomics. Biotechnol Adv. 2014;32:122-36.

15. Cápal P, Blavet N, Vrána J, Kubaláková M, Doležel J. Multiple displacement amplification of the DNA from single flow-sorted plant chromosome. Plant J. 2015. doi:10.1111/tpj.13035.

16. Vrána J, Kubaláková M, Č́íhalíková J, Valárik M, Doležel J. Preparation of sub-genomic fractions enriched for particular chromosomes in polyploid wheat. Biol Plant. 2015;59:445-55.

17. Vrána J, Kubaláková M, Šimková H, Číhalíková J, Lysák MA, Doležel J. Flow sorting of mitotic chromosomes in common wheat (Triticum aestivum L.) Genetics. 2000;156:2033-41.

18. Giorgi D, Farina A, Grosso V, Gennara A, Ceoloni C, Lucretti S. FISHIS: fluorescence in situ hybridization in suspension and chromosome flow sorting made easy. PLoS One. 2013;8:e57994.

19. Kubaláková M, Kovářová P, Suchánková P, Číhalíková J, Bartoš J, Lucretti S, Watanabe N, Kianian SF, Doležel J. Chromosome sorting in tetraploid wheat and its potential for genome analysis. Genetics. 2005;170:823-9. 